\title{
Weak tangent and level sets of Takagi functions
}

\author{
Han $Y u^{1}$
}

Received: 28 November 2018 / Accepted: 27 January 2020 / Published online: 1 February 2020

(c) The Author(s) 2020

\section{Abstract}

In this paper, we study some properties of Takagi functions and their level sets. We show that for Takagi functions $T_{a, b}$ with parameters $a, b$ such that $a b$ is a root of a Littlewood polynomial, there exist large level sets. As a consequence, we show that for some parameters $a, b$, the Assouad dimension of graphs of $T_{a, b}$ is strictly larger than their upper box dimension. In particular, we can find weak tangents of those graphs with large Hausdorff dimension, larger than the upper box dimension of the graphs.

Keywords Assouad dimension · Littlewood polynomial · Level sets of Takagi functions

Mathematics Subject Classification Primary: 28A80 · 37C45; Secondary: 26A27

\section{Introduction}

In this paper, we study graphs of the following functions,

$$
T_{a, b}(x)=\sum_{n=0}^{\infty} a^{n} T\left(b^{n} x\right),
$$

where $a, b$ are real parameters $a, b$ such that $a<1, b>1, a b \geq 1$ and $T: \mathbb{R} \rightarrow \mathbb{R}$ is the tent map which has period 1 and defined on the unit interval as follows,

$$
T(x)= \begin{cases}x & x \in\left[0, \frac{1}{2}\right] \\ 1-x & x \in\left[\frac{1}{2}, 1\right] .\end{cases}
$$

Communicated by Adrian Constantin.

Han Yu

hy351@cam.ac.uk

1 Department of Pure Mathematics and Mathematical Statistics, University of Cambridge, Cambridge CB3 0WB, UK 
Such functions $T_{a, b}$ are called Takagi functions. Originally the Takagi function was referred to as $T_{1 / 2,2}$ but no confusion should appear if we also call $T_{a, b}$ to be a Takagi function. There has been a lot of interest in the Hausdorff and box counting dimensions of the graphs of such functions. For the box dimensions we know from [14, Section 2] and [4, Theorem 2.4] that the upper box dimension of graphs of these functions $T_{a, b}$ can be computed by the following formula

$$
B=2+\frac{\ln a}{\ln b}=1+\frac{\ln a b}{\ln b} .
$$

The Hausdorff dimensions of graphs of these functions are harder to obtain, see [19], [5] and the references therein for more recent results on related questions.

One of the results of this paper is about the Assouad dimension of some Takagi functions. In what follows, for a function $f: \mathbb{R} \rightarrow \mathbb{R}$, we denote the following set

$$
\Gamma_{f}=\left\{(x, y) \in \mathbb{R}^{2}: x \in[0,1], y=f(x)\right\},
$$

to be the graph of $f$ over the interval $[0,1]$.

Theorem 1.1 (Assouad dimension) Let $a, b$ be positive numbers and the product $a b>$ 1 be a root of a Littlewood polynomial of degree $k-1$, namely

$$
\sum_{n=0}^{k-1} \epsilon_{n}(a b)^{n}=0
$$

for a sequence $\left\{\epsilon_{n}\right\}_{n \in\{0, \ldots, k-1\}}$ over $\{-1,1\}$. Furthermore, if $b$ is an integer greater than 2, then we have the following result,

$$
\operatorname{dim}_{\mathrm{A}} \Gamma_{T_{a, b}} \geq 1+\frac{1}{k}
$$

Remark 1.2 In fact, the proof of this theorem shows that

$$
\operatorname{dim}_{\mathrm{A}}^{1 / B} \Gamma_{T_{a, b}} \geq 1+\frac{1}{k}
$$

where $B=\ln \left(a b^{2}\right) / \ln b$ and $\operatorname{dim}_{\mathrm{A}}^{1 / B}$ is the Assouad spectrum with parameter $1 / B$. In this paper, we focus only on the Assouad dimension. For more details on Assouad spectrum, see [10].

Notice that by keeping the product $a b$ unchanged and making $b$ larger, this lower bound can be larger than the upper box dimension $\frac{\ln a b^{2}}{\ln b}$ for large $b$. For example, when we choose parameters such that $a b=\frac{\sqrt{5}+1}{2}, b=8$, then $\overline{\operatorname{dim}_{\mathrm{B}}} \Gamma_{T_{a, b}} \approx 1.23$ and $\operatorname{dim}_{\mathrm{A}} \Gamma_{T_{a, b}} \geq 4 / 3$.

One consequence of Theorem 1.1 is that there exist large weak tangents of the graphs of Takagi functions. See Sect. 4 for more details about the notions of dimensions, the definition of weak tangent and some basic properties. 
Lemma 1.3 (Weak tangent) Let $a, b$ be as in the statement of Theorem 1.1, then there exists a weak tangent $E$ of $\Gamma_{T_{a, b}}$ such that

$$
\operatorname{dim}_{\mathrm{H}} E=\operatorname{dim}_{\mathrm{A}} \Gamma_{T_{a, b}} \geq \overline{\operatorname{dim}_{\mathrm{B}}} \Gamma_{T_{a, b}}
$$

The rightmost inequality can be strict.

Theorem 1.1 follows from the existence of a large level set of graphs $\Gamma_{T_{a, b}}$ and we think this result is interesting on its own.

Theorem 1.4 Let $T_{a, b}$ be as in the statement of Theorem 1.1 but we also allow $a b=1$. For each $y \in \mathbb{R}$ we define the following level set

$$
L(y)=\left\{x \in[0,1]: T_{a, b}(x)=y\right\} \times\{y\} .
$$

There exists $y \in \mathbb{R}$ such that

$$
\operatorname{dim}_{\mathrm{H}} L(y) \geq \frac{1}{k}
$$

The restriction of the product $a b$ as a certain type of algebraic integers seems to be strong. However, with some effort, we can show that those algebraic integers are dense in $\left[\frac{1}{2}, 2\right]$.

Theorem Let $L$ be the set of algebraic integers which are roots of Littlewood polynomials namely, $x \in \mathbb{C}$ and there exist an integer $k \geq 1$ and a finite sequence $\epsilon_{n} \in\{ \pm 1\}, n \in\{0, \ldots, k-1\}$ such that

$$
\sum_{n=0}^{k-1} \epsilon_{n} x^{n}=0 .
$$

Then $L \cap\left[\frac{1}{2}, 2\right]$ is dense in $\left[\frac{1}{2}, 2\right]$.

Proofs of the above result and its generalizations can be found in [2,3,18].

\section{Discussions and future work}

In this section, we give some backgrounds of Theorems 1.1 and 1.4. We also pose some questions which are related to the results in this paper.

\subsection{Assouad dimensions of graphs of functions}

Theorem 1.1 deals with the Assouad dimension of some Takagi functions. It is natural to think about the Assouad dimension of other nowhere differentiable functions, for example, Weierstrass functions and graphs of the Wiener process. For the latter, we have the following result ([12, Theorem 2.2]). 
Theorem 2.1 (HY17) The graph of the Wiener process $W($.$) over the unit interval has$ Assouad dimension 2 almost surely.

We have not completely determined the Assouad dimension of any Takagi function yet. We only showed that the Assouad dimension could be strictly larger than the upper box dimension for graphs of Takagi functions.

Question 2.2 For $a, b \in \mathbb{R}^{+}$and $a b>1$, determine the Assouad dimension for the graph of $T_{a, b}$.

\subsection{Level sets of Takagi functions}

For more details about the level sets of Takagi functions, see $[1,16]$. Notice that if we set $a=0.5, b=2$ then we can find level set of $T_{a, b}$ with Hausdorff dimension at least 0.5 . This is sharp, see [6]. For other values of $a, b$ for example $a=(\sqrt{5}+1) / 16, b=8$ we see that we can find a level set with Hausdorff dimension at least $1 / 3$ and we do not know whether this is sharp.

Question 2.3 What is the largest Hausdorff dimension achieved by the level sets of the Takagi function $T_{\frac{\sqrt{5}+1}{16}, 8}$ ?

\section{Notation}

1. For a real number $x \in \mathbb{R}$ we use the symbol $x^{+}$to denote a number $x+\epsilon$ where $\epsilon>0$ is some fixed positive number whose value can be chosen freely and we will point out the specific value of $\epsilon$ when necessary. Similarly, we use $x^{-}$for a number smaller but close to $x$.

2. For a function $f: \mathbb{R} \rightarrow \mathbb{R}$, the following set

$$
\Gamma_{f}=\left\{(x, y) \in \mathbb{R}^{2}: x \in[0,1], y=f(x)\right\},
$$

is called the graph of $f$ over the interval $[0,1]$.

3. For a real number $x$, we use $\lfloor x\rfloor$ to denote the greatest integer that is not strictly larger than $x$.

\section{Preliminaries}

We will now introduce some notions of dimensions which will be used in this paper. We use $N_{r}(F)$ for the minimal covering number of a bounded set $F$ in $\mathbb{R}^{n}$ with balls of side length $r>0$. 


\subsection{Hausdorff dimension}

The Hausdorff dimension of $F$ is defined to be

$$
\operatorname{dim}_{\mathrm{H}} F=\inf \left\{s: \forall \delta>0, \exists\left\{U_{i}\right\}_{i=1}^{\infty} \text { such that } \bigcup_{i} U_{i} \supset F, \sum_{i} \operatorname{diam}\left(U_{i}\right)^{s}<\delta\right\} .
$$

\subsection{Upper box dimension}

The upper box dimension of $F$ is

$$
\overline{\operatorname{dim}_{\mathrm{B}}} F=\limsup _{r \rightarrow 0}\left(-\frac{\log N_{r}(F)}{\log r}\right) .
$$

\subsection{Assouad dimension and weak tangents}

The Assouad dimension of $F$ is

$$
\begin{array}{r}
\operatorname{dim}_{\mathrm{A}} F=\inf \{s \geq 0:(\exists C>0)(\forall R>0)(\forall r \in(0, R))(\forall x \in F) \\
\left.N_{r}(B(x, R) \cap F) \leq C\left(\frac{R}{r}\right)^{s}\right\}
\end{array}
$$

where $B(x, R)$ denotes the closed ball of centre $x$ and radius $R$.

An important tool for studying the Assouad dimension is weak tangents introduced in [17] and microsets in [11]. The next definition appeared in [9, Definition 1.1].

Definition 4.1 Let $X \in \mathcal{K}\left(\mathbb{R}^{n}\right)$ be a fixed reference set (usually the closed unit ball or cube) and let $E, F \subset \mathbb{R}^{n}$ be compact sets. Suppose there exists a sequence of similarity maps $T_{k}: \mathbb{R}^{n} \rightarrow \mathbb{R}^{n}$ such that $d_{\mathcal{H}}\left(E, T_{k}(F) \cap X\right) \rightarrow 0$ as $k \rightarrow 0$. Then $E$ is called a weak tangent of $F$.

Here $\left(\mathcal{K}\left(\mathbb{R}^{n}\right), d_{\mathcal{H}}\right)$ is a complete metric space with the Hausdorff metric, namely, for two compact subsets $A, B \subset \mathbb{R}^{n}$ is defined by

$$
d_{\mathcal{H}}(A, B)=\inf \left\{\delta>0: A \subset B_{\delta}, B \subset A_{\delta}\right\}
$$

where for any compact set $C \subset \mathbb{R}^{n}$

$$
C_{\delta}=\left\{x \in \mathbb{R}^{n}:|x-y|<\delta \text { for some } y \in C\right\} .
$$

Lemma 1.3 is a direct consequence of Theorem 1.1 and the following result, see [15, Proposition 5.7]. 
Theorem 4.2 (KOR) Let $F$ be a compact set with $\operatorname{dim}_{\mathrm{A}} F=s$. Then there exist a weak tangent $E$ of $F$ such that

$$
\operatorname{dim}_{\mathrm{H}} E=s
$$

In other words, we have

$$
\operatorname{dim}_{\mathrm{A}} F=\max \left\{\operatorname{dim}_{\mathrm{H}} E: E \text { is a weak tangent of } F\right\}
$$

\subsection{Covering by disjoint cubes}

For convenience, in this paper we will count covering number with disjoint squares rather than balls. We denote $S(a, R)$ for $a \in \mathbb{R}^{2}, R>0$ as the square centred at $a$ with side length $2 R$ whose sides are parallel to the coordinate axis. Since we are dealing with graph of functions, the choice of axis is natural. We denote the following covering number,

$$
\begin{aligned}
& N(F \cap S(a, R), r)=\mid\left\{(i, j) \in \mathbb{Z}^{2} \cap[0,\lfloor R / r\rfloor+1]^{2}:\right. \\
& S((a-R / 2+r / 2+i r, a-R / 2+r / 2+j r), r) \cap F \neq \emptyset\} \mid .
\end{aligned}
$$

This is equivalent to $N_{r}(F \cap B(a, R))$ in the sense that there exists a constant $C>0$ such that for all $a \in F, 0<r<R<1$ we have the following inequality,

$$
C^{-1} N_{r}(F \cap B(a, R)) \leq N(F \cap S(a, R), r) \leq C N_{r}(F \cap B(a, R))
$$

\subsection{Some properties of Takagi functions}

In this paper, we will use the following result whose proof can be found in [13], and we use the version presented in [4, Theorem 2.4].

Lemma 4.3 Let $T: \mathbb{R} \rightarrow \mathbb{R}$ be a continuous piecewise $C^{1}$ and periodic function. Then the following function

$$
T_{a, b}(x)=\sum_{n=0}^{\infty} a^{n} T\left(b^{n} x\right)
$$

must satisfy either one of the two properties,

1: $T_{a, b}$ is piecewise $C^{1}$.

2: For a positive constant $C>0$ and any interval $J \subset \mathbb{R}$ we have the following inequality,

$$
\sup _{x, y \in J}\left|T_{a, b}(x)-T_{a, b}(y)\right| \geq C|J|^{-\frac{\ln a}{\ln b}}
$$


Notice that if $a<1, a b>1$ then $-\frac{\ln a}{\ln b} \in(0,1)$ and we see that if $|J|<1$ then

$$
\sup _{x, y \in J}\left|T_{a, b}(x)-T_{a, b}(y)\right| \geq C|J| \text {. }
$$

Remark 4.4 When $T$ is the tent map defined in the beginning of the first section, it is known that when $a<1, a b \geq 1$, the function $T_{a, b}$ is nowhere differentiable therefore only the second property of Lemma 4.3 can be true.

\section{Large level sets, proof of Theorem 1.4}

We show that there exist large level sets for function $T_{a, b}$ with certain parameters $a, b$. Since $a b$ is a root of a Littlewood polynomial we see that

$$
\sum_{i=0}^{k} \epsilon_{i}(a b)^{i}=0
$$

for an integer $k \geq 1$ and some choice of $\epsilon_{i} \in\{ \pm 1\}$. Next we consider the first $k$ terms partial sum

$$
F_{1}(x)=\sum_{n=0}^{k-1} a^{n} T\left(b^{n} x\right) .
$$

The derivative of the above function is not continuous at $x=m b^{-k}$ for integers $m$. Let us now assume that $x$ is an irrational number then the derivative is

$$
F_{1}^{\prime}(x)=\sum_{i=0}^{k-1} \epsilon_{i}(x)(a b)^{i},
$$

where $\epsilon_{i}(x) \in\{ \pm 1\}$ depends on the $b$-nary expansion of $x$. In particular, if

$$
x=0 . b_{1} b_{2} \ldots
$$

then

$$
\epsilon_{i}(x)= \begin{cases}1 & b_{i} \in[0, b / 2] \\ -1 & b_{i} \in(b / 2,1] .\end{cases}
$$

Therefore we can find at least 2 disjoint intervals of length $\frac{1}{2 b^{k-1}}$ where $F_{1}^{\prime}(x)=0$ and $F_{1}(x)=a_{1}$ is for a constant $a_{1} \geq 0$ on those two intervals. Indeed, when

$$
\sum_{i=0}^{k-1} \epsilon_{i}(a b)^{i}=0
$$


we also have

$$
-\sum_{i=0}^{k-1} \epsilon_{i}(a b)^{i}=0
$$

So there are at least two intervals we can find and the union is symmetric with respect to the line $\{x=0.5\}$. Then because $F_{1}$ is also symmetric with respect to the line $\{x=0.5\}$ we see that $F_{1}($.$) takes the same value on those two intervals, say, I_{1}$ and $I_{2}$.

We consider the next $k$ terms sum

$$
F_{2}(x)=\sum_{n=k}^{2 k-1} a^{n} T\left(b^{n} x\right) .
$$

Then we can find $b$ many intervals of length $1 / 2 b^{2 k-1}$ in $I_{1}, I_{2}$ such that the above sum stays constant $a_{2} \geq 0$ on those intervals. To see this, consider $I_{1}$, which is an interval of length $1 / 2 b^{k}$. Now observe the following

$$
F_{2}(x)=\sum_{n=k}^{2 k-1} a^{n} T\left(b^{n} x\right)=\sum_{n=0}^{k} a^{n+k} T\left(b^{k} b^{n} x\right)=a^{k} F_{1}\left(b^{k} x\right) .
$$

Therefore the graph of $F_{2}$ is an affine copy, or intuitively speaking, a narrowed version of the graph of $F_{1}$. Then we see that there are exactly $b$ many intervals in $I_{1}$ of length $1 / 2 b^{2 k-1}$ such that $F_{2}$ equals to $a_{2}$ on all those intervals. Indeed, over any interval the form $\left[l / b^{k-1},(l+1) / b^{k-1}\right]$ the graph of $F_{2}$ has $2 b$ many plateaux of the same level. That is to say, we can find $2 b$ many $1 / 2 b^{2 k-1}$ length intervals on which $F_{2}(x)=a_{2}$. Since $I_{1}$ is only a half $1 / b^{k-1}$ length interval, therefore we can find $b$ many plateaux over $I_{1}$. Here we used the mirror symmetry of $F_{2}$.

We can apply the above argument to $j$-th $k$-terms partial sums for each $j \geq 2$ and as a result we can find a Cantor set $C$ such that $T_{a, b}(C)=\{c\}$ for a constant $c$. By construction, this Cantor set is obtained by first taking two intervals $I_{1}, I_{2}$ of length $1 / 2 b^{k-1}$ then placing $b$-many intervals of length $1 / 2 b^{2 k-1}$ inside of each $I_{1}, I_{2}$. Then, inside of those intervals of length $1 / 2 b^{2 k-1}$, we place $b$ many intervals of length $1 / 2 b^{3 k-1}$. This procedure goes on indefinitely and self-similarly (just like the construction of the middle third Cantor set). As a result, the obtained Cantor set is a self-similar set satisfying the open set condition and its Hausdorff dimension is $1 / k$ (the contraction ratio is $1 / b^{k}$ and branching number is $b$, see for example [7, Theorem 9.3]). Thus we have proved Theorem 1.4.

\section{Squashing and counting, proof of Theorem 1.1}

In order to deal with the Assouad dimension of graphs of Takagi functions we need to handle the following quantity

$$
N\left(S(x, R / 2) \cap \Gamma_{\left.T_{a, b}, r\right) .}\right.
$$


The situation is not too bad when we want to deal with the above quantity for $\Gamma_{f+g}$ when one of the functions, say $f$, is Lipschitz continuous. The following result is a localized and quantitative version of [8, Lemma 2.1,2.2].

Lemma 6.1 If $f:[0,1] \rightarrow \mathbb{R}$ is Lipschtz continuous with Lipschitz constant $M>0$ and $g:[0,1] \rightarrow \mathbb{R}$ is continuous then we have the following inequality for $0<r<$ $R<1$ whenever $\frac{R}{r}$ is an integer,

$$
\begin{aligned}
& \sup _{a \in[0,1] \times \mathbb{R}} N\left(S(a, R / 2) \cap \Gamma_{f+g}, r\right) \\
& \geq \frac{1}{M+2} \sup _{a \in[0,1] \times \mathbb{R}} N\left(S(a, R / 2) \cap \Gamma_{g}, r\right)-\frac{M+2}{\lfloor M\rfloor+2} \frac{R}{r} .
\end{aligned}
$$

Remark 6.2 For the case when $R / r$ is not an integer, we can replace $r$ with a larger value

$$
r^{\prime}=R \frac{1}{\lfloor R / r\rfloor}
$$

As we will eventually choose $R / r$ to be arbitrarily large, $r^{\prime}$ and $r$ are essentially the same. For any $\delta>0$, if $R / r$ is large enough the following relation holds

$$
(1-\delta) r<r^{\prime}<(1+\delta) r
$$

Then the inequality of this theorem holds with $1 /(M+2)$ being replaced by some other constant which depends only on $M$.

Proof of Lemma 6.1 Let $a \in \mathbb{R}^{2}$ and consider the square $S(a, R / 2)$. For any $r<R$ we consider the following rectangles for $i=0,1, \ldots, \frac{R}{r}-1$

$$
S_{i}=[a-R / 2+i r, a-R / 2+(i+1) r] \times[a-R / 2, a+R / 2] \subset S(a, R / 2) .
$$

Each rectangle $S_{i}$ contains the following squares for $j=0,1, \ldots, \frac{R}{r}-1$

$$
S_{i j}=[a-R / 2+i r, a-R / 2+(i+1) r] \times[a-R / 2+j r, a-R / 2+(j+1) r] .
$$

Now if $S_{i j} \cap \Gamma_{g} \neq \emptyset$ we colour it black, otherwise we colour it white. Let $n_{i} \geq 0$ denote the number of black squares among $S_{i j}, j=0,1,2, \ldots, \frac{R}{r}-1$. By continuity of $g$, the fact that we have $n_{i}$ black squares implies the following inequality

$$
\sup _{x, y \in[a-R / 2+i r, a-R / 2+(i+1) r]}|g(x)-g(y)| \geq\left(n_{i}-2\right) r .
$$

By Lipschitz property of function $f$ we see that

$$
|f(x)-f(y)| \leq M|x-y|
$$


this implies that

$$
\sup _{x, y \in[a-R / 2+i r, a-R / 2+(i+1) r]}|f(x)-f(y)| \leq M r .
$$

Then we see that

$$
\sup _{x, y \in[a-R / 2+i r, a-R / 2+(i+1) r]}|f(x)+g(x)-f(y)-g(y)| \geq\left(n_{i}-2-M\right) r .
$$

So we see that to cover the set

$$
\left\{(x, y+f(x)) \in \mathbb{R}^{2}:(x, y) \in S_{i} \cap \Gamma_{g}\right\}
$$

we need at least

$$
n_{i}-2-M
$$

many squares of side length $r$. Summing over all $i$ we see that to cover the set

$$
\left\{(x, y+f(x)) \in \mathbb{R}^{2}:(x, y) \in S(a, R / 2) \cap \Gamma_{g}\right\}
$$

we need at least

$$
\sum_{i} n_{i}-(M+2) \frac{R}{r}
$$

many squares with side length $r$.

The next fact to notice is that the following set

$$
\left\{(x, y+f(x)) \in \mathbb{R}^{2}:(x, y) \in S(a, R / 2) \cap \Gamma_{g}\right\}
$$

is contained in a $R \times(M+1) R$ rectangle. This rectangle can be covered by $\lfloor M\rfloor+2$ squares with side length $R$, so for at least one of the $\lfloor M\rfloor+2$ squares need at least

$$
\frac{\sum_{i} n_{i}-(M+2) \frac{R}{r}}{\lfloor M\rfloor+2}
$$

many squares with side length $r$ to cover.

It is then easy to see that

$$
\left\{(x, y+f(x)) \in \mathbb{R}^{2}:(x, y) \in S(a, R / 2) \cap \Gamma_{g}\right\} \subset \Gamma_{f+g} .
$$

This implies that

$$
\sup _{a^{\prime} \in[0,1] \times \mathbb{R}} N\left(S\left(a^{\prime}, R / 2\right) \cap \Gamma_{f+g}, r\right) \geq \frac{\sum_{i} n_{i}-(M+2) \frac{R}{r}}{\lfloor M\rfloor+2},
$$


and since $\frac{R}{r}$ is integer we see that

$$
\sum_{i} n_{i}=N\left(S(a, R / 2) \cap \Gamma_{g}, r\right) .
$$

It follows that

$\sup _{a^{\prime} \in[0,1] \times \mathbb{R}} N\left(S\left(a^{\prime}, R / 2\right) \cap \Gamma_{f+g}, r\right) \geq \frac{1}{M+2} N\left(S(a, R / 2) \cap \Gamma_{g}, r\right)-\frac{M+2}{\lfloor M\rfloor+2} \frac{R}{r}$,

We can take the supreme of $a$ on the right hand side of the above inequality and the lemma concludes.

Now we can move on to dealing with the Assouad dimension of Takagi functions. We shall need the following lemma.

Lemma 6.3 For any number $1<\beta<2$ and all integers $k$, there exists a sequence $\epsilon_{n}$ of \pm 1 such that $\left|\sum_{n=0}^{k} \epsilon_{n} \beta^{n}\right| \leq \frac{1}{\beta-1}$.

Proof Let $k>0$ be an integer, consider the following two functions forming an IFS (known as iterated function system, see for example [7, chapter 9]),

$$
f_{-1}(x)=\beta x-1, f_{1}(x)=\beta x+1 \text {. }
$$

Then for any sequence $\epsilon_{n} \in\{ \pm 1\}$ with $n=0,1,2, \ldots, k-1$ we can define an iteration by

$$
f_{\epsilon_{0}} \circ \cdots \circ f_{\epsilon_{k-2}} \circ f_{\epsilon_{k-1}}
$$

and notice that

$$
f_{\epsilon_{0}} \circ \cdots \circ f_{\epsilon_{k-2}} \circ f_{\epsilon_{k-1}}(0)=\sum_{n=0}^{k-1} \epsilon_{n} \beta^{n}
$$

So as long as we can find an iteration of this IFS such that the trajectory of 0 stays bounded by $\frac{1}{\beta-1}$ the existence of a sequence $\epsilon_{n}$ will follow. Now since $f_{1}(0)=1<$ $\frac{1}{\beta-1}$ which is the intersection of the line $y=f_{1}(x)$ and $y=x$, we can apply function $f_{1}$ before the value exceeds $\frac{1}{\beta-1}$ and apply $f_{-1}$ before the value drops below $-\frac{1}{\beta-1}$. More precisely, we put $x_{0}=0$ and if we find $x_{i} \in(-1 /(\beta-1), 1 /(\beta-1))$ then if $f_{1}\left(x_{i}\right)<1 /(\beta-1)$ we set $x_{i+1}=f_{1}\left(x_{i}\right)$ otherwise we set $x_{i+1}=f_{-1}\left(x_{i}\right)$. We need to check $x_{i+1} \in(-1 /(\beta-1), 1 /(\beta-1))$ as well. In fact, if $x_{i+1}>1 /(\beta-1)$ then $f_{-1}\left(x_{i}\right)>1 /(\beta-1)$ and this implies that $x_{i}>1 /(\beta-1)$. If $x_{i}<-1 /(\beta-1)$ then either $f_{1}\left(x_{i}\right)<-1 /(\beta-1)$ or $f_{-1}\left(x_{i}\right)<-1 /(\beta-1)$ in the first case we have $x_{i}<-1 /(\beta-1)$. The latter case implies that $x_{i}<(2-\beta) / \beta(\beta-1)$ but then $f_{1}\left(x_{i}\right)<1 /(\beta-1)$. So in any case $x_{i+1} \in(-1 /(\beta-1), 1 /(\beta-1))$ as required. This 
procedure gives us an sequence $\epsilon_{n}$, with $n=0,1,2, \ldots, k-1$ for any integer fixed $k-1$ such that

$$
\left|\sum_{n=0}^{k-1} \epsilon_{n} \beta^{n}\right| \leq \frac{1}{\beta-1}
$$

Notice that when $\beta$ is a root of a Littlewood polynomial, it is necessary that $0.5 \leq$ $|\beta| \leq 2$. Therefore if parameters of $T_{a, b}$ are as stated in Theorem 1.1 then $1 \leq a b \leq 2$ and therefore the result of Lemma 6.3 holds for $a b$. Now we have all the ingredients needed to prove Theorem 1.1 however, we find it convenient to introduce the following general result.

Lemma 6.4 (Squash and count) Suppose $T_{a, b}(x): \mathbb{R} \rightarrow \mathbb{R}$ is a function of the following form

$$
T_{a, b}(x)=\sum_{n=0}^{\infty} a^{n} T\left(b^{n} x\right)
$$

where $T(x): \mathbb{R} \rightarrow \mathbb{R}$ is a piecewise $C^{1}$ continuous function with period 1 and $a>0, b>0, a b>1$. Suppose the following two conditions holds:

1, (interval with slow changing): There exists a positive constant $C_{1}>0$ such that for any integer $M>0$, there is a integer $k$ such that $J_{k}=\left(\frac{k}{b^{M+1}}, \frac{k+1}{b^{M+1}}\right)$ and for all $x_{1}, x_{2} \in J_{k}$ the following condition holds,

$$
\left.\left.\mid \sum_{n=0}^{M} a^{n} T\left(b^{n} x_{1}\right)\right)-\sum_{n=0}^{M} a^{n} T\left(b^{n} x_{2}\right)\right)\left|<C_{1}\right| x_{1}-x_{2} \mid .
$$

2, (large level set): There exists a level set $L \subset[0,1]$ with lower box dimension at least $D$, namely,

$$
\exists y \in \mathbb{R}, \underline{\operatorname{dim}_{\mathrm{B}}} L(y)=\underline{\operatorname{dim}_{\mathrm{B}}}\left\{x \in[0,1]: T_{a, b}(x)=y\right\} \geq D .
$$

Then we have the following result,

$$
\operatorname{dim}_{\mathrm{A}} \Gamma_{T_{a, b}} \geq D+1
$$

Proof For any positive integer $M>0$, we can find an integer $k$ and $x_{0}=\frac{k}{b^{M+1}}$ such that $\left(x_{0}, x_{0}+\frac{1}{b^{M+1}}\right) \subset[0,1]$ and on this subset we have the following condition for the oscillation of the following $M$-th partial sum

$$
\left.\left.\mid \sum_{n=0}^{M} a^{n} T\left(b^{n} x_{1}\right)\right)-\sum_{n=0}^{M} a^{n} T\left(b^{n} x_{2}\right)\right)\left|<C_{1}\right| x_{1}-x_{2} \mid,
$$


where $x_{1}, x_{2} \in\left(x_{0}, x_{0}+\frac{1}{b^{M+1}}\right)$. Then we can write that

$$
\begin{aligned}
T_{a, b}(x) & =\sum_{n=0}^{\infty} a^{n} T\left(b^{n} x\right)=\sum_{n=0}^{M} a^{n} T\left(b^{n} x\right)+\sum_{n=M+1}^{\infty} a^{n} T\left(b^{n} x\right) \\
& =F_{M}(x)+G_{M}(x),
\end{aligned}
$$

where the functions $F_{M}, G_{M}$ are the first sum and second sum in the third expression. Then we see that the graph $\Gamma_{G_{M}}$ is actually a 'squashed' version of $\Gamma_{T_{a, b}}$, namely we have the following relation

$$
X_{M}\left(\Gamma_{T_{a, b}}\right)=\Gamma_{G_{M}},
$$

where the linear transformation $X_{M}: \mathbb{R}^{2} \rightarrow \mathbb{R}^{2}$ is defined to be as follows

$$
X_{M}(x, y)=\left(\frac{x}{b^{M+1}}, a^{M+1} y,\right) .
$$

We see that since $a b>1$ this linear transformation squashes a square to a very thin rectangle for large enough $M$. Now we concentrate on the strip

$$
S=\left(x_{0}, x_{0}+\frac{1}{b^{M+1}}\right) \times \mathbb{R} .
$$

The graph $\Gamma_{G_{M}}$ over this strip is the squashed version of the graph $\Gamma_{T_{a, b}}$ over [0, 1$]$. We want to find a $\frac{1}{b^{M+1}}$-square contained in this strip such that we need a reasonably large amount of $r$-squares to cover $\Gamma_{G_{M}}$, where $r>0$ is number that will be specified later. Now because of the bijective linear map $X_{M}$, covering a $\frac{1}{b^{M+1}}$-square with $r$ square in $\Gamma_{G_{M}}$ is the same thing as covering the original graph $\Gamma_{T_{a, b}}$ over $[0,1]$ inside a $1 \times \frac{1}{(a b)^{M+1}}$-rectangle with $r b^{M+1} \times \frac{r}{a^{m+1}}$-rectangles.

Now consider the level set $L$ with lower box dimension $D$ mentioned in the second condition. For some real number $y^{\prime}$ we have that

$$
L=L\left(y^{\prime}\right)=\left\{x \in[0,1]: T_{a, b}(x)=y^{\prime}\right\} \times\left\{y^{\prime}\right\}
$$

then we do box counting in the $1 \times \frac{1}{(a b)^{M+1}}$-rectangle containing $L$. For any $\left(x, y^{\prime}\right) \in L$ the graph $\Gamma_{T_{a, b}}$ intersects the middle axis of the rectangle at $\left(x, y^{\prime}\right)$, so by Lemma 4.3 we see that there exists a positive constant $C_{3}>0$ such that the projection of graph $\Gamma_{T_{a, b}}$ inside any $\frac{1}{(a b)^{M+1}}$-square centred in $L$ to the vertical axis has length at least

$$
\min \left(C_{3} \frac{1}{(a b)^{M+1}}, \frac{1}{(a b)^{M+1}}\right) .
$$

If $M$ is large enough we need at least $\left(\frac{1}{a b}\right)^{-D^{-}(M+1)}$ many $\frac{1}{(a b)^{M+1}}$-square to cover this $1 \times \frac{1}{(a b)^{M+1}}$-rectangle because for covering the level set $L$ we already need that 
many squares. Since each square is sufficiently occupied by $\Gamma_{T_{a, b}}$ in the sense that the curve occupies at least $\min \left(C_{3}, 1\right)$ portion of the vertical length. This means that for each such square we need some constant times

$$
\frac{1 /(a b)^{M+1}}{r / a^{M+1}}=\frac{1}{r b^{M+1}}
$$

many $r b^{M+1} \times \frac{r}{a^{m+1}}$-rectangle to cover. Now we choose the following value for $r$

$$
r=\frac{1}{\left(a b^{2}\right)^{M+1}}=\left(\frac{1}{(a b)^{M+1}}\right)^{\frac{\ln a b^{2}}{\ln a b}}=\left(\frac{1}{(a b)^{M+1}}\right)^{\frac{B}{B-1}}=\left(\frac{1}{b^{M+1}}\right)^{B}
$$

where $B=2+\frac{\ln a}{\ln b}$ is the upper box dimension of $\Gamma_{T_{a, b}}$. We denote $R=\frac{1}{b^{M+1}}$ and note that $r=R^{\frac{1}{\theta}}$ with $\theta=\frac{1}{B}$ and we get the following relation

$$
\sup _{a \in \mathbb{R}^{2}} N\left(S(a, R / 2) \cap \Gamma_{G_{M}}, R^{\frac{1}{\theta}}\right) \geq\left(\frac{1}{a b}\right)^{-D^{-}(M+1)} \frac{1}{r b^{M+1}}=\left(\frac{R}{r}\right)^{1+D^{-}}
$$

The above inequality holds for arbitrarily large $M$ and therefore it holds also for arbitrarily small $R, R^{\frac{1}{\theta}}$. This is a covering property for $\Gamma_{G_{M}}$ and we can translate it to a covering property for $\Gamma_{T_{a, b}}$. By using Lemma 6.1 and Remark 6.2 together with condition (1) we see that there exist a constant $C>0$ such that

$$
\begin{aligned}
& \sup _{a \in\left[x_{0}, x_{0}+\frac{1}{b^{M+1}}\right] \times \mathbb{R}} N\left(S(a, R / 2) \cap \Gamma_{T_{a, b}}, r\right) \\
& \geq C \sup _{a \in\left[x_{0}, x_{0}+\frac{1}{b^{M+1}}\right] \times \mathbb{R}} N\left(S(a, R / 2) \cap \Gamma_{G_{M}}\right)-C \frac{R}{r} \\
& \geq C\left(\frac{R}{r}\right)^{1+D^{-}}-C \frac{R}{r} .
\end{aligned}
$$

Then by definition of the Assouad dimension, we see that

$$
\operatorname{dim}_{\mathrm{A}} \Gamma_{T_{a, b}} \geq 1+D
$$

This concludes the proof.

We can now finish the proof of Theorem 1.1. By Lemma 6.4 and Theorem 1.4 we see that it is enough to show that the Takagi functions satisfy condition (1) in the statement of Lemma 6.4. In fact, condition (1) is satisfied by $T_{a, b}$ whenever $a<$ $1, b \in\{2\} \cup[2, \infty], a b \in(1,2)$. We put the last step of proving Theorem 1.1 in the following lemma. 
Lemma 6.5 If the parameters $a, b$ satisfy the following conditions

$$
a<1, b \in\{2\} \cup[3, \infty], 2>a b>1,
$$

then the Takagi functions

$$
T_{a, b}(x)=\sum_{n=0}^{\infty} a^{n} T\left(b^{n} x\right)
$$

satisfy the condition 1 in the statement of Lemma 6.4.

Proof For each integer $M>0$ we shall consider the $M$-level intervals

$$
I_{M}(j)=\left[\frac{j}{2 b^{M}}, \frac{j+1}{2 b^{M}}\right], j \in \mathbb{Z} .
$$

If $b \geq 3$, we see that

$$
\frac{1}{2 b^{M}} \geq \frac{3}{2 b^{M+1}},
$$

this implies that any interval $I_{M}(j)$ contains at least two $(M+1)$-level intervals say

$$
I_{M+1}(l), I_{M+1}(l+1) \text {. }
$$

$l, l+1$ is a pair of integers, one of them is odd and the other is even. It is easy to see that this is also true for $b=2$. Then we see that given any sequence $\omega_{i} \in\{ \pm 1\}, i=$ $0,1,2 \ldots$ it is possible to choose a sequence of integers $j_{i}, i=0,1, \ldots$ such that

$$
I_{i}\left(j_{i}\right) \subset I_{i-1}\left(j_{i-1}\right) \subset[0,1],
$$

and that $j_{i}$ is even if and only if $\omega_{i}=1$. We see that

$$
T^{\prime}\left(b^{M} x\right)= \begin{cases}1 & x \in I_{M}(j), j \text { is even } \\ -1 & x \in I_{M}(j), j \text { is odd }\end{cases}
$$

So for any integer $M>0$ we can find intervals $I_{M}(j) \subset[0,1]$ such that

$$
\left.D_{M}(x)=\sum_{n=0}^{M} a^{n} T\left(b^{n} x\right)\right)^{\prime}=\sum_{n=0}^{M} a^{n} b^{n} T^{\prime}\left(b^{n} x\right)
$$

is constant on $I_{M}(j)$ and the value can take all numbers in the following set

$$
U=\left\{t \in \mathbb{R}: \exists \epsilon_{n} \in\{ \pm 1\}, n \in\{0,1,2 \ldots, M\}, t=\sum_{n=0}^{M} \epsilon_{n}(a b)^{n}\right\}
$$

Then by Lemma 6.3 we see that the result follows. 
Acknowledgements HY was financially supported by the Corpus Christi College, Cambridge. HY has received funding from the European Research Council (ERC) under the European Union's Horizon 2020 research and innovation programme (Grant Agreement No. 803711). This paper was written when HY was a Ph.D. student at the University of St Andrews. HY thanks Jon Fraser for discussions and comments.

Open Access This article is licensed under a Creative Commons Attribution 4.0 International License, which permits use, sharing, adaptation, distribution and reproduction in any medium or format, as long as you give appropriate credit to the original author(s) and the source, provide a link to the Creative Commons licence, and indicate if changes were made. The images or other third party material in this article are included in the article's Creative Commons licence, unless indicated otherwise in a credit line to the material. If material is not included in the article's Creative Commons licence and your intended use is not permitted by statutory regulation or exceeds the permitted use, you will need to obtain permission directly from the copyright holder. To view a copy of this licence, visit http://creativecommons.org/licenses/by/4.0/.

\section{References}

1. Allaart, P., Kawamura, K.: The Takagi function: a survey. Real Anal. Exch. 37, 1-54 (2011)

2. Baker, S., Yu, H.: Root sets of polynomials and power series with finite choices of coefficients. Comput. Methods Funct. Theory 18, 89-97 (2018)

3. Bandt, C.: On the Mandelbrot set for pairs of linear maps. Nonlinearity 15, 1127-1147 (2002)

4. Barański, K.: Dimension of the Graphs of the Weierstrass-Type Functions, pp. 77-91. Springer, Cham (2015)

5. Barański, K., Bárány, B., Romanowska, J.: On the dimension of the graph of the classical Weierstrass function. Adv. Math. 265, 32-59 (2014)

6. de Amo, E., Bhouri, I., Díaz Carrillo, M., Fernández-Sánchez, J.: The Hausdorff dimension of the level sets of Takagi's function. Nonlinear Anal. Theory Methods Appl. 74, 5081-5087 (2011)

7. Falconer, K.: Fractal Geometry: Mathematical Foundations and Applications, 2nd edn. Wiley, New York (2005)

8. Falconer, K., Fraser, J.: The horizon problem for prevalent surfaces. Math. Proc. Camb. Philos. Soc. 151, 355-372 (2011)

9. Fraser, J.: Distance sets, orthogonal projections, and passing to weak tangents. Isr. J. Math. 226(2), 851-875 (2018)

10. Fraser, J., Yu, H.: New dimension spectra: finer information on scaling and homogeneity. Adv. Math. 329, 273-328 (2018)

11. Furstenberg, H.: Ergodic fractal measures and dimension conservation. Ergod. Theory Dyn. Syst. 28, 405-422 (2008)

12. Howroyd, D., Yu, H.: Assouad dimension of random processes. Proc. Edinb. Math. Soc. 62(1), 281-290 (2019)

13. Hu, T.Y., Lau, K.S.: Fractal dimensions and singularities of the Weierstrass type functions. Trans. Am. Math. Soc. 335, 649-665 (1993)

14. Kaplan, J., Mallet-Paret, J., Yorke, J.: The Lyapunov dimension of a nowhere differentiable attracting torus. Ergod. Theory Dyn. Syst. 4(2), 261-281 (1984)

15. Käenmäki, A., Ojala, T., Rossi, E.: Rigidity of quasisymmetric mappings on self-affine carpets. International Mathematics Research Notes, preprint, arXiv:1607.02244 (to appear)

16. Lagarias, J.: The Takagi function and its properties. RIMS Kokyuroku Bessatsu B34, 153-189 (2012)

17. Mackay, J.M., Tyson, J.T.: Conformal Dimension. Theory and Application, University Lecture Series, vol. 54. American Mathematical Society, Providence, RI (2010)

18. Odlyzko, A., Poonen, B.: Zeros of polynomials with 0,1 coefficients. Enseign. Math. 39, 317-348 (1993)

19. Shen, W.X.: Hausdorff dimension of the graphs of the classical Weierstrass functions. Math. Z. 2017, $1-44$ (2017)

Publisher's Note Springer Nature remains neutral with regard to jurisdictional claims in published maps and institutional affiliations. 